\title{
Water droplet erosion of aeroengine fan blades: the importance of form
}

\author{
Charles B. Burson-Thomas ${ }^{\mathrm{a}, *}$, Richard Wellman ${ }^{\mathrm{b}}$, Terry J. Harvey ${ }^{\mathrm{a}}$, Robert J.K. Wood ${ }^{\mathrm{a}}$ \\ ${ }^{a}$ National Centre for Advanced Tribology at Southampton (nCATS), University of Southampton, United Kingdom \\ ${ }^{b}$ Surface Engineering, Rolls-Royce plc., United Kingdom
}

\begin{abstract}
The radius of typical water droplet, which an aeroengine encounters in-service, and the radius of curvature of a typical fan blade leading-edge are approximately the same; this is unlike previously investigated contexts, where the typical droplet radius is at least an order of magnitude smaller. The consequences of this have been explored theoretically but never experimentally. In this study, an established technique was used to generate high-speed impingements $\left(210 \mathrm{~m} \mathrm{~s}^{-1}\right.$ to $\left.250 \mathrm{~m} \mathrm{~s}^{-1}\right)$ of water on planar and curved (in one plane) samples of a comparatively well-understood material (polymethylmethacrylate). A novel, direct, characterisation methodology (using ultra-high-speed imaging) was employed to accurately describe each impingement. The form of damage changed significantly when a curved sample was subjected to a high-speed impingement; the circular damage pattern observed on planar samples changed to an oval. This is qualitatively consistent with the theoretical predictions and constitutes another step forward in the understanding of the WDE of aeroengine fan blades.
\end{abstract}

Keywords: Water Droplet Erosion, Fan blades, Ultra-high-speed imaging, Liquid impingement

\section{Introduction}

The Water Droplet Erosion (WDE) of fan blades, on large civil aeroengines, can lead to a decrease in engine performance and increase the risk of unstable vibrations [1]. Despite the undesirable effects of this form of wear, the WDE of aeroengine fans has received little attention and few descriptions exist in the literature; just one reference to fans [2] has been found in our review of decades of literature on WDE. Instead, it has been the WDE of steam turbines and the leading surfaces of aircraft that has driven much of the research effort over the years 3 . However, there is a fundamental difference between these contexts and the WDE of aeroengine fan blades; the radius of curvature of the fan blade leading-edge (approx. $1 \mathrm{~mm}$ ) and typical droplet radius $(0.5 \mathrm{~mm}$ to $2.5 \mathrm{~mm}$ ) are of the same order of magnitude [4. Extension of the analytical theoretical analyses, to include a solid surface with a radius of curvature in one plane (rather than assumed to be planar), has predicted a different

\footnotetext{
${ }^{*}$ Corresponding author

Email address: c.bursonthomas@soton.ac.uk (Charles B. Burson-Thomas)
}

loading, and severity of mechanisms, for a highspeed water droplet impingement on the leadingedge of an aeroengine fan blade; asymmetric, as opposed to axisymmetric [4]. Although there have been previous investigations into WDE with tests conducted on curved samples [5], an in-depth experimental study of the implications of the radius of curvature has not been performed. In order to verify the theoretical work and further investigate the importance of form in the WDE of aeroengine fan blades, a new experimental facility at University of Southampton has been developed and a testing programme is under way. In this paper, this new facility is described, including a novel, direct methodology for characterising the impinging water, as well as the results of testing conducted on curved samples. The ultimate goal is to explore the effect of form, in the early stages of WDE, with in-service material systems, e.g. titanium alloy (Ti-6Al-4V), and potential in-service material systems, e.g. alloys with a surface coating and/or surface modification. However, at this stage it is only possible to share the results from initial tests using PMMA (polymethylmethacrylate), primarily selected as its response (under high-speed impingement) is com- 
paratively well-understood.

Water Droplet Erosion is a specific case of 'liquid impingement erosion', which has been defined as: "progressive loss of original material from a solid surface due to continued exposure to impacts by liquid drops or jets" 6]. When the research surrounding WDE is initially viewed, it can be difficult to see a consistent picture; the mechanisms of material removal are often described in material and situation specific terms, such is the scope of many studies. There is also the absence of a complete theory of WDE, under which to unite the field 7]. Finally, the response of the material is often strongly influenced by the 'severity' of the repeated impingements; although not perfect, the impingement velocity offers a good indicator in this regard. Note that for aeroengine fan-blades, impingement velocities of $200 \mathrm{~m} \mathrm{~s}^{-1}$ to $400 \mathrm{~m} \mathrm{~s}^{-1}$ are expected [4. Despite these challenges, a general classification of erosion mechanisms has been proposed [8] and offers a helpful framework around which to appreciate how repeated high-speed impingement leads to WDE. However, before these are described, it is first necessary to explain the process of a high-speed droplet impingement, which drives the mechanisms.

A high-speed, $50 \mathrm{~m} \mathrm{~s}^{-1}$ and above [9], droplet impingement is often described as a two-stage process 3, 10. This is shown in Fig. 1 and described below:

(a) Initial 'compressible' stage. When the droplet first contacts the solid surface, it behaves compressibly 11 and a region of compressed liquid within the droplet, at the interface with the solid surface, is formed. This is caused by an initial expansion of the contact periphery, between the liquid droplet and the surface, that is more rapid than the motion of the shock front. The compressed liquid exerts very high pressures (order of hundreds of $\mathrm{MPa}$ for aeroengine fan blades), often described as the 'water-hammer' pressure, on the solid surface during this stage.

(b) Secondary 'flow' stage. Very shortly after the start of the impingement (less than a $\mu s$ ), the shock front is able to overtake the contact periphery and the release of the compressed liquid can commence. This takes the form of high-velocity flows of liquid in a radial direction. The velocity of the lateral jets are significantly higher than the impingement velocity, with some measurements suggesting a value up to ten times greater [12]. Along with the radial

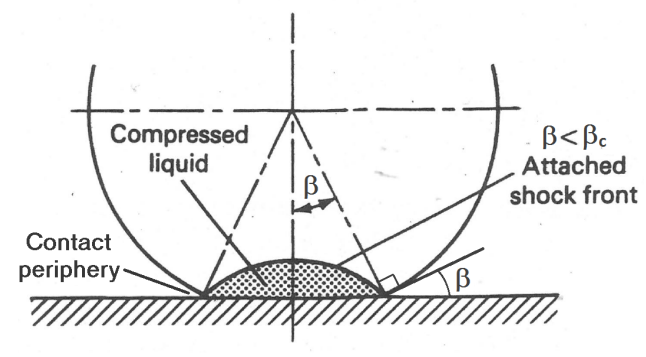

(a) Initial compressible stage, the shaded region is compressed liquid.

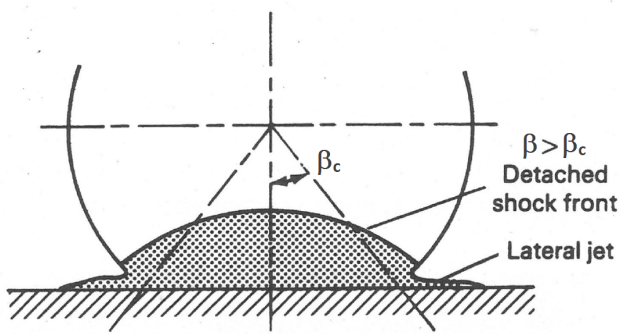

(b) Secondary flow stage, which can commence once shock front overtakes contact periphery.

Figure 1: Two stages of high-speed droplet impingement (adapted from [10]).

jets, release waves propagate from the contact periphery to the central axis, leading to a substantial drop in pressure (at least an order of magnitude) to the Bernoulli stagnation pressure.

The four general erosion mechanisms that result from repeated high-speed water droplet impingement on a solid surface are:

(i) Direct deformation. This describes the effect of the high 'water-hammer' pressure, applied to the surface of the material during the compressible stage. The nature of the material is key in determining how this dynamic and transient loading results in damage and the subsequent erosion. Typically, in the early stages of the WDE of a ductile metal (assuming the surface is initially smooth), large numbers of small surface depressions are formed ('pitting') [13. By contrast, when the loading is applied to brittle materials, both surface and sub-surface damage results due to tensile failure [8].

(ii) Stress wave propagation. The high pressures 
applied during the compressible stage also results in the propagation of stress waves (compression, shear, and Rayleigh [8]) through the solid. These stress waves can interact with features within the material, reinforce, or reach a boundary and reflect, resulting in regions of tensile loading that can lead to damage and erosion [14].

(iii) Lateral outflow jetting. The high-velocity lateral jets, produced during the flow stage, can interact with irregularities on the solid surface, leading to plastic deformation and crack propagation [8]. The nature of the surface and velocity of jets are thought to be the key drivers of the severity of this mechanism [15].

(iv) Hydraulic penetration. This mechanism describes the effect of the change in surface geometry of the solid, resulting from the establishment of an erosion pit or crack, causing stress concentrations. These result in the accelerated propagation of cracks, which tunnel under the surface of the material and result in the upheaval of the overlying material [16].

The lateral outflow jetting and hydraulic penetration mechanisms require a surface discontinuity or pre-existing pit in order to be active [8]. Therefore, if the solid surface is initially smooth, direct deformation and stress wave propagation will be the only mechanisms active in the early stages ('incubation' period [13]) of WDE. Once the initially smooth and crack-free leading-edges of a titanium alloy aeroengine fan blade begin to become damaged by the action of impinging water droplets, and all four mechanisms become active, the engineering solutions to prevent further erosion are limited. Therefore, if seeking to prevent or minimise WDE on aeroengine fan blades, a prudent approach is to prevent or extend the time before the initial surface discontinuities form (the precursors to wear) [4].

Thus, our research has focussed on understanding the direct deformation and stress wave propagation erosion mechanisms in the context of aeroengine fan blades. Both of these mechanisms are a result of the initial compressible stage only. This point is fundamental to the theoretical analysis previously published [4] and the experimental effort described in this paper; in order to understand the implications, for the stage of WDE that is of most importance in terms of engineering solutions for aeroengine fan blades, we need only consider the initial compressible stage of a water droplet impingement.
This paper begins by describing and justifying the material selected to verify the theoretical predictions, as well as detailing the experimental methodology employed and the state-of-the-art imaging equipment that was used to make direct measurements of the impinging water, which has not previously been possible. Following this, a theoretical justification for the analysis made from the sets of images taken with the ultra-high-speed camera is provided. Then some key results of experimental testing so far are presented and subsequently discussed. Finally, conclusions are offered.

\section{Material and methods}

\subsection{Material selection}

In order to understand how the radius of curvature of the surface influences the mechanisms by which a high-speed water droplet causes damage, it is prudent to initially use a material whose response is well-understood. PMMA (polymethylmethacrylate) has been used extensively in previous WDE research 14, 17, 18, 19, 20, 21, 22, 23. In addition to the damage mechanisms resulting from the highspeed impingement of water being comparatively well-understood, it is also: inexpensive, transparent, readily machinable, and has low minimum impingement velocity (less than $150 \mathrm{~m} \mathrm{~s}^{-1}$ ) for visible damage 23 . Thus, it was selected as the first material to explore the effect of solid surface form with.

As might be expected, the response of PMMA to an high-speed water droplet impingement is dependent on impingement velocity 21. As the velocity increases, the form of damage changes, e.g. a depressed annular region becomes a ring crack, and new damage modes are introduced. Therefore, when discussing the expected response of the PMMA it is essential to refer to the response at the correct range of impingement velocities. As will be shown later, the experimental set-up generated high-speed impingements with a velocity between 210 and $250 \mathrm{~m} \mathrm{~s}^{-1}$. At impingement velocities below $400 \mathrm{~m} \mathrm{~s}^{-1}$, Adler 21 describes the following characteristic features of damage: a central undamaged region, surrounded by a smooth depressed annular region, which, in turn, is surrounded by short circumferential crazing fractures that extend to distances greater than the radius of the impinging droplet. Figure 2 shows how these features present in a light micrograph. For the sample shown in this figure, the depth of the annular depression ranged between 200 and $400 \mathrm{~nm}$ [19]. 


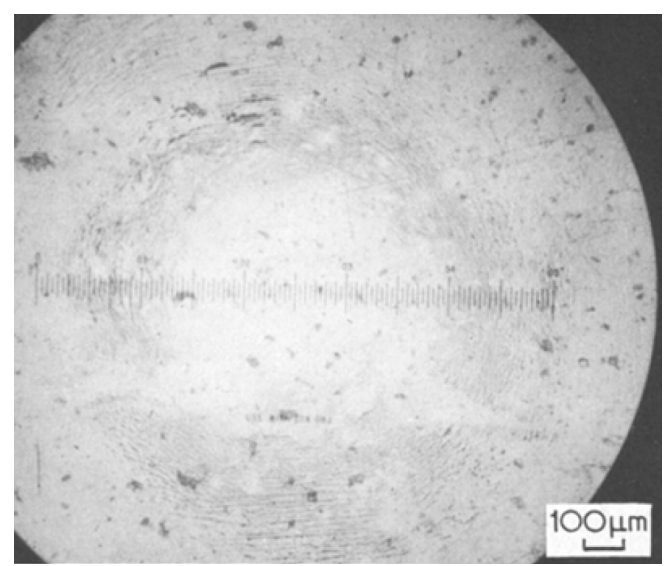

Figure 2: Typical annular circumferential crack pattern on PMMA for impingement velocities of interest. Specimen was impinged by a water droplet with a diameter of $1.8 \mathrm{~mm}$ at $222 \mathrm{~m} \mathrm{~s}^{-1} 19$.

As well as determining the characteristic features of damage, the impingement velocity also determines their dimensions [20]. For example, as the impingement velocity increases, so does the diameter of central undamaged region. The droplet radius also affects the dimensions of damage features. It is thought that for a given impingement velocity, key dimensions describing the damage, normalised by the droplet radius, remain constant; if a droplet of double the radius impinges a surface at the same velocity, the damage pattern is identical, just twice the size [20]. A number of studies have investigated the ability to predict the dimensions of the characteristic damage based on impingement velocity and droplet radius [19, 20, 21]. The radius of the central undamaged region, particularly when measured using a profilometer 21, enjoys strong correlation with predictions from analytical theory. Different criteria have been proposed to develop the analytical prediction, however, as Adler [8] shows, the difference between the two is minimal for impingement velocities below $700 \mathrm{~m} \mathrm{~s}^{-1}$. What emerges is that the edge of the central undamaged region, and so its radius, is strongly correlated to the point where the shock front overtakes the contact periphery.

\subsection{Experimentally simulating the compressible stage}

In order to subject a solid surface to a loading akin to the compressible stage, an impingement of water at high-speed is required. Generating this is not trivial and a number of different approaches

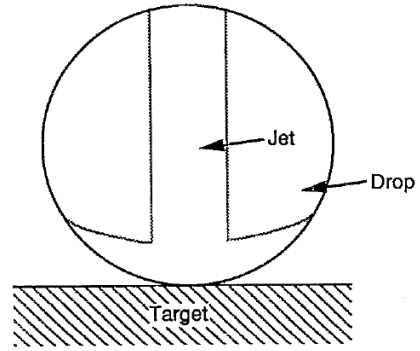

Figure 3: Ideal form of high-speed curved-fronted jet 23.

have been employed by investigators in the past 7]. In this study, a high-speed curved-fronted water-jet is used to recreate the impingement of a droplet. This technique has been instrumental in developing understanding of WDE and used successfully for over 50 years [24, 25]. The theoretical justification for using jets to recreate the impingement of a droplet is conveniently summarised in Fig. 3. If this ideal case occurs, the compressible stage of this jet and and a spherical droplet (of the same radius and velocity) would be identical [23].

However, studies employing high-speed photography [20, 23. have shown that the ideal case does not quite occur in practice; air ablation results in a jet with a curved front but with a varying radius of curvature. Previously, in order to determine the equivalent droplet diameter for a given experimental condition, empirical correlations were established using the same material that is the subject of this study (PMMA). Initially, the diameter of the central undamaged region for jet and droplet impingement were compared [20]. Hand et al. 23] developed the methodology to use area (instead of the radius) of the central undamaged region for this purpose. However, recent advances in ultra-highspeed digital imaging (up to $5 \mathrm{MHz}$ ) mean that it is now possible to measure the surface profile of the impinging high-speed water jet directly. This has been used in this study and is described later.

\subsection{Test rig design and experimental procedure}

Figure 4 shows a schematic of the experimental set-up. The impact of a $9 \mathrm{~mm}$ chrome steel (AISI 52100 ) ball, accelerated by a gas-gun, is used to produce the high-speed water jet. The acceleration of the chrome steel ball was highly repeatable during the tests; the mean ball velocity close to the barrel exit was $55.4 \mathrm{~m} \mathrm{~s}^{-1}$ with a standard deviation of $0.7 \mathrm{~m} \mathrm{~s}^{-1}$. To produce the high-speed jets, the 


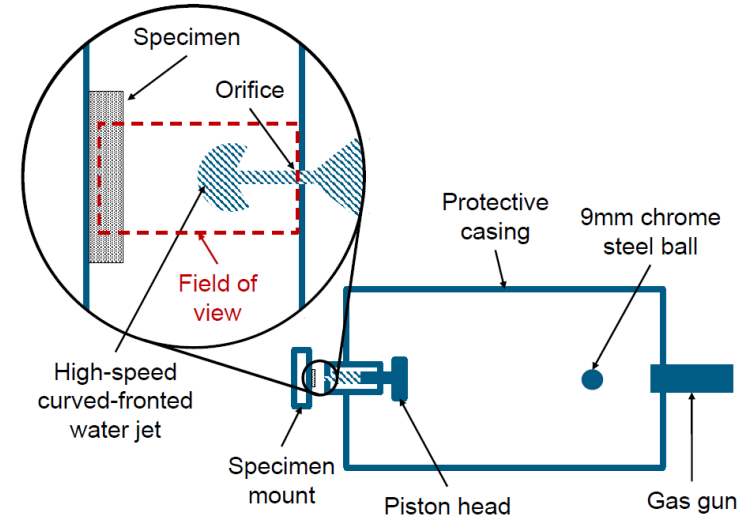

Figure 4: Experimental set-up for experimentally simulating a high-speed droplet impingement on a specimen.

chamber of a specially-designed nozzle (see Hand et al. 23] for geometry) is filled with water, covered with a small piston at the rear, which (when impacted by the ball) produces a jet from an orifice. An ultra-high-speed camera, a Shimadzu HPV-X2, was used to record the motion and geometry of the jet, allowing the velocity and form to be measured directly. Planar and curved samples were mounted at an appropriate stand-off distance from the orifice. Figure 5 shows both planar and curved samples about to be impinged by a high-speed jet.

Two light-gates, located close to the exit of the gas gun, were used to measure the velocity of the $9 \mathrm{~mm}$ ball and trigger the ultra-high-speed camera and flash. It was necessary to trigger the flash in advance of jet production as it takes approximately $120 \mu$ s for the flash, a Bowens Gemini 1000 Pro, to reach peak output after receiving the trigger signal. However, if a typical jet is considered, average velocity of $200 \mathrm{~m} \mathrm{~s}^{-1}$ across the stand-off distance of $10 \mathrm{~mm}$, it will take only $50 \mu \mathrm{s}$ to reach the sample. Thus, the output from each of the two light gates was connected to an Arduino microcontroller, which used the time delay between the two signals to calculate the velocity of the ball and from this predict when the ball will impact the top of the piston. By using appropriate delays it was possible to trigger the flash and then camera, $100 \mu \mathrm{s}$ later, to capture the high-speed jet as it traversed the stand-off. The ultra-high-speed camera (Shimadzu HPV-X2) records a total of 128 frames, thus when running at the frame rate selected, $500 \mathrm{kHz}$, the total recording length was $256 \mu \mathrm{s}$, providing a useful margin for error in the triggering.

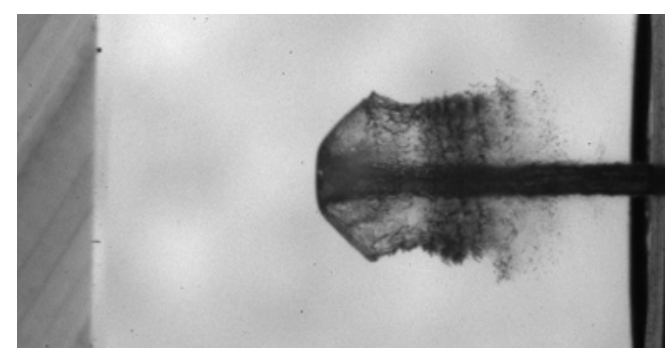

(a) Impinging a planar surface

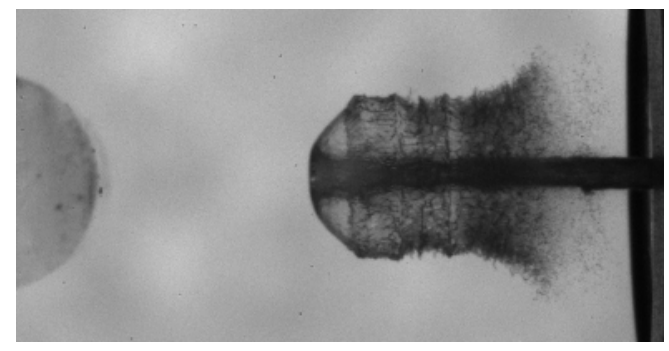

(b) Impinging a curved surface, radius of curvature of $2 \mathrm{~mm}$

Figure 5: Examples of high-speed curved-fronted water jets impinging samples.

Table 1 summaries the experimental parameters. All tests were conducted at room temperature. The stand-off distance, type of water used, and surface preparation are consistent with previous investigations [14, 21, 24]. The surface of the PMMA must be sufficiently smooth for it to be transparent (a key property for many users), thus no additional surface preparation was required beyond that performed by the supplier. Roughness measurements (in accordance with ISO 4288:1996), using a Taylor-Hobson Talysurf-120L, of 'as recieved' planar and curved sample surfaces gave mean values of arithmetic average roughness $\left(R_{a}\right)$ of $10 \mathrm{~nm}$ and $13 \mathrm{~nm}$ respectively. Given the localisation of damage close to the impinged surface at velocities below $400 \mathrm{~m} \mathrm{~s}^{-1}$ 21 it was possible to use PMMA rod to act as surface with a radius of curvature (in one plane), as the rear side of the rod, to that presented to the high-speed jet, was not expected to influence results. In addition, the lateral dimension(s) of the samples $(25 \mathrm{~mm}$ for the rod and $25 \mathrm{~mm} \times 25 \mathrm{~mm}$ for the planar) meant they could be considered to be semi-infinite. The PMMA used for the planar specimens was Perspex cell cast acrylic sheet, machined to the appropriate dimensions; extruded acrylic rod was used for the curved samples, cut to the appro- 
Table 1: Summary of experimental parameters selected

\begin{tabular}{ll}
\hline Parameter & Value \\
\hline Stand-off distance & approx. 10 mm \\
Water & Tap water \\
Surface preparation & As received \\
Dimensions of planar sam- & $5 \mathrm{~mm} \times 25 \mathrm{~mm} \times 25 \mathrm{~mm}$ \\
ples & \\
Length of curved samples & $25 \mathrm{~mm}$ \\
Radii of curved samples & $1,1.5,2,2.5,3,4$, \\
& 5,6, and $7.5 \mathrm{~mm}$ \\
Gas gun pressure & $140 \mathrm{kPa}$ \\
Orifice diameter & $0.6 \mathrm{~mm}$ \\
\hline
\end{tabular}

Table 2: Properties of PMMA material used for planar and curved samples 26, 27.

\begin{tabular}{lll}
\hline Property & $\begin{array}{l}\text { Planar } \\
\text { (Cast } \\
\text { Perspex) }\end{array}$ & $\begin{array}{l}\text { Curved } \\
\text { (Extruded } \\
\text { Acrylic) }\end{array}$ \\
\hline Density & $1.19 \mathrm{~g} \mathrm{~cm}^{-3}$ & $1.18 \mathrm{~g} \mathrm{~cm}^{-3}$ \\
Elastic Modulus & $3210 \mathrm{MPa}$ & $3300 \mathrm{MPa}$ \\
Tensile Strength & $75 \mathrm{MPa}$ & unavailable \\
Poisson's Ratio & 0.38 & unavailable \\
\hline
\end{tabular}

priate length. Table 2, 26, 27] shows some of the key material properties of each type of PMMA used. The gas gun pressure and orifice diameter selected were the result of a initial set of characterisation tests; this combination reliably produced coherent, stable jets of the desired velocity and curvature.

\subsection{Post-experimental analysis}

Once the experiment had been run and the images recorded, the program MATLAB was used to perform the processing. The following list describes the steps in the image processing protocol.

1. Select the relevant images from the 128-frame recording.

2. Find the difference of the value at each pixel, in each frame, with value at each pixel in the subsequent frame ('frame difference').

3. Apply the edge-detection algorithm by Trujillo-Pino et al. 28] to each frame difference, to track the jet front with sub-pixel accuracy.
4. Calculate the average velocity between two frame differences by taking the change in position between the point on jet front furthest from orifice in each and dividing by inter-frame time. As the inter-frame time is small, this value approximates the instantaneous velocity at the point in time halfway between the two frame differences.

5. Select the points identified by edge-detection algorithm within $0.2 \mathrm{~mm}$ of the point on jet front furthest from orifice. Fit, using method of least-squares, an appropriate function (see next section for detail) to describe form of jet front on each frame difference. This was done in two stages, where initially the function was fitted to all the points within $0.2 \mathrm{~mm}$ in order to determine the jet centre. Then, the points within one orifice diameter $(0.6 \mathrm{~mm})$ above and below the centre line were selected and the final fit performed to these points only.

In order to convert between pixels and metres, calibration images were taken at appropriate intervals. The values of velocity and jet form considered to be the values on impingement were the last value of each quantity preceding contact between the jet and surface. Finally, an Olympus BX-51 optical microscope was used to produce the light micrographs and a TaiCaan XYris 4000 CL to perform the 3D profilometry scans. Removal of surface form, for both the planar and curved samples, was performed using the TaiCaan 'BODDIES' software.

\section{Theory}

As previously described, in this study, direct measurements of the impinging high-speed water jets were made in order to characterise each impingement by measuring the velocity and form. After analysing the images from the ultra-high-speed camera it was clear that (as has been previously observed [23]), whilst the jets were curved, they did not have a constant radius of curvature. A secondorder polynomial (or quadratic) function was found to better describe a plane through the axisymmetric jet front. Therefore, the next step was to understand how a coherent volume of liquid, with an axisymetric front described by a rotated quadratic function, impinges a solid surface and how that relates to a droplet impingement.

This question has been resolved by employing the fundamental assumptions and approach of 'Guided 


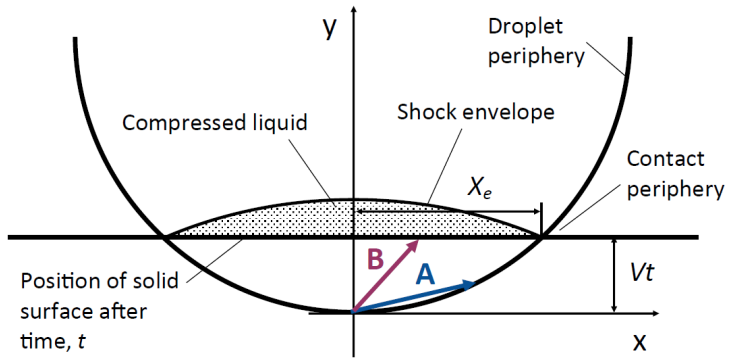

Figure 6: Spherical droplet impinging a planar surface after time, $t$. The coordinate system is fixed to the original position of the droplet.

Acoustic Shock', as described in Lesser 99. It has been shown, for the compressible stage of a highspeed droplet impingement, the effect of viscosity and surface tension are minimal and it is the inertial effects that drive the process [29, 30. By considering only the dominant physics and assuming the solid surface to be rigid, it has been possible to develop a simple relationship between the coefficient of the second-order term in the quadratic expression describing the form of the jet (the $a$ in $\left.y=a x^{2}\right)$ and the radius of a droplet that would produce an 'equivalent' loading during the compressible stage. The underlying principle that has been used to establish this 'equivalence' is impulse (the integral of force with respect to the time for which it acts); what droplet would impart the same impulse during the compressible stage as the measured high-speed jet.

An alternative mathematical method, to that used in previous work, was used to arrive at the necessary relationships describing the motion of the contact periphery. This was the use of vector calculus. In order to demonstrate the validity of the approach, and explain the process by which it is employed, the first subsection will show how it can be used to derive the well-known equations for droplet impingement. The next subsection will use this same approach to derive the equations for the simplified jet front, a coherent volume of water with an axisymetric front described by a rotated quadratic function. Finally, the third subsection uses the equality of impulse to derive the relationship that allows an 'equivalent radius' to be calculated from the second-order coefficient in the quadratic describing the form of the jet.

\subsection{Vectorial methodology to derive equations}

The equations describing the initial kinematics of a liquid droplet impingement were first proposed by Bowden and Field [11. A step-by-step derivation of these expressions is provided in Engel [2] or Adler 8. Figure 6 shows 2D plane through the axisymmetric situation presented by a spherical droplet impinging a planar surface. The coordinate system is fixed with respect to the droplet, thus at time, $t$ the solid surface has moved by a distance $V t$, where $V$ is the impingement velocity, in the positive $\mathbf{y}$-direction. Two vectors, $\mathbf{A}$ and $\mathbf{B}$ are defined, where $\mathbf{A}$ is a position vector from the origin (point of initial contact) to any point on the liquid droplet periphery; $\mathbf{B}$ is a position vector from the origin to any point on the solid surface. $\hat{\mathbf{i}}$ and $\hat{\mathbf{j}}$ are unit vectors in the $\mathrm{x}$ and $\mathrm{y}$ directions respectively. The expression for $\mathbf{A}$ (eqn. 1) has been constructed using the equation for a circle with radius, $r$ and a centre located at $(0, r)$.

$$
\begin{gathered}
\mathbf{A}=(x) \hat{\mathbf{i}}+\left(\left(r^{2}-x^{2}\right)^{1 / 2}+r\right) \hat{\mathbf{j}} \\
\mathbf{B}=(x) \hat{\mathbf{i}}+(V t) \hat{\mathbf{j}}
\end{gathered}
$$

At the contact periphery $\mathbf{A}=\mathbf{B}$. If the coefficients of $\hat{\mathbf{j}}$ in eqn. 1 and 2 are equated and rearranged, the well-known [2, 8, equation for the radial position of the contact periphery $\left(X_{e}\right)$, when the contact area over which the high-pressures associated with the initial compressible stage is expanding, is found.

$$
X_{e}=x=\left(2 r V t-(V t)^{2}\right)^{1 / 2}
$$

A new position vector can be defined, $\mathbf{C}$, from the origin to the contact periphery. This a function of: droplet radius, $r$; impingement velocity, $V$; and time, $t$.

$$
\mathbf{C}=\left(2 r V t-(V t)^{2}\right)^{1 / 2} \hat{\mathbf{i}}+(V t) \hat{\mathbf{j}}
$$

The condition for the point at which the shock front overtakes the contact periphery, when the contact area over which the high-pressures associated with the initial compressible stage starts to contract, is suggested to be $|\dot{\mathbf{C}}|=C_{1}$ [11, where $C_{1}$ is the shock speed in the liquid. If eqn. 4 is differentiated with respect to time, an expression for $\dot{\mathbf{C}}$ is found.

$$
\dot{\mathbf{C}}=\frac{V(r-V t)}{\left(2 r V t-(V t)^{2}\right)^{1 / 2}} \hat{\mathbf{i}}+(V) \hat{\mathbf{j}}
$$


If the modulus of eqn. 5 is calculated, the wellknown equation for of the radial position where lateral outflow jetting is predicted to commence, $X_{c}=V r / C_{1}$, by Bowden and Field [11] is found.

$$
|\dot{\mathbf{C}}|=\sqrt{\left(\frac{V(r-V t)}{\left(2 r V t-(V t)^{2}\right)^{1 / 2}}\right)^{2}+V^{2}}=\frac{V r}{x}
$$

Thus, a vectorial approach offers a convenient and accurate alternative methodology to derive key equations for liquid droplet impingement.

\subsection{Impingement of jet with surface described by second order polynomial}

In this subsection, the vectorial methodology is applied to understand the impingement conditions where a coherent volume of liquid, shaped in the form of an elliptic paraboloid (a quadratic rotated around its axis of symmetry), impinges a solid, planar surface. As this situation is also axi-symmetric, it can be simplified to the $2 \mathrm{D}$ case of a parabola, described by a second order polynomial $y=a x^{2}$, impinging a planar surface, represented by a straight line. The coordinate system continues to be fixed to the liquid, thus the previous expression for $\mathbf{B}$ (eqn. 2) remains the same. However, a new expression for $\mathbf{A}$ is required due to the change of form, where $a$ is the coefficient of the second-order term.

$$
\mathbf{A}=(x) \hat{\mathbf{i}}+\left(a x^{2}\right) \hat{\mathbf{j}}
$$

As previously, if the coefficients of $\hat{\mathbf{j}}$ are equated and re-arranged, this time for eqn. 7 and 2 , an equation for the radial position of the contact periphery $\left(X_{e}\right)$ is found.

$$
X_{e}=x=\sqrt{\frac{V t}{a}}
$$

The position vector, $\mathbf{C}$, which is now a function of $a, V$ and $t$, can be defined from the origin to the contact periphery.

$$
\mathbf{C}=\left(\frac{V t}{a}\right)^{1 / 2} \hat{\mathbf{i}}+(V t) \hat{\mathbf{j}}
$$

Applying the Bowden and Field [11] condition, via calculating $\dot{\mathbf{C}}$ from eqn. 9 , then $|\dot{\mathbf{C}}|$, and finally equating to $C_{1}$, leads to an expression for the time at which the shock front overtakes the contact periphery, $t_{c}$.

$$
t_{c}=\frac{V}{4 a\left(C_{1}^{2}-V^{2}\right)}
$$

\subsection{Equivalent droplet radius}

As described in the introduction to this section, the impulse of the impinging liquid was been used to generate an expression for the equivalent droplet radius. The desired expression will be of the form $r=f(a)$, where $a$ is the coefficient of the secondorder term in the quadratic expression $\left(y=a x^{2}\right)$, where this second-order polynomial is used to describe the axisymmetric high-speed jet front. The expressions derived in the previous sub-sections are used in order to derive this. Impulse $(J)$, the integral of force $(F)$ with respect to the time of application (from $t_{1}$ to $t_{2}$ ), is described mathematically as:

$$
J=\int_{t_{1}}^{t_{2}} F d t
$$

During the compressible stage, when the highpressures are applied, the magnitude of the pressure is often assumed to be constant and uniform [3. The 'water-hammer' pressure $(p)$, is calculated using eqn. 12, which is commonly attributed 31] to Cook $32 . \rho_{1}$ is the density of the liquid.

$$
p=\rho_{1} C_{1} V
$$

Whilst the pressure is considered time-invariant, the area of application $(A)$ is not; it varies and can be sub-divided into two phases of the compressible stage [4]:

(1) 0 to $t_{c}$ The contact periphery is expanding, leading to a circular area of application, which is increasing in size, with a radius of $X_{c}$. This expansion continues until the shock front has overtaken the contact periphery, when $t=t_{c}$ and $X_{e}=X_{c}$.

(2) $t_{c}$ to $t_{r}$ The circular area of application, with a radius of $X_{r}$, is now contracting as the release wave propagates from the contact periphery to the central axis. When $t=t_{r}$ the compressible stage is complete.

Calculating the total impulse of the compressible stage is most conveniently done by considering the two phases separately and then summing. This is 
shown in the equation below, where $p \pi r^{2}$ has been substituted for $F$.

$$
J=p \pi \int_{0}^{t_{c}} X_{e}^{2} d t+p \pi \int_{t_{c}}^{t_{r}} X_{r}^{2} d t
$$

In order to solve eqn. 13 for a jet front described by a quadratic rotated around its axis of symmetry and sphere of liquid, both impinging a planar surface, expressions for $X_{e}, X_{r}, t_{c}$, and $t_{r}$ are required for each. As previously described, for a sphere of liquid on a planar surface, these expressions have been available for many decades (see Engel [2] or Adler [8]). For the jet-front described by a quadratic (rotated around its axis of symmetry), eqn. 8 gives $X_{e}$ and eqn. 10 gives $t_{c}$. Therefore, expressions for $X_{r}$ and $t_{r}$ still needed to be found. However, before this is described, a useful approximation of eqn. 10 is made, the expression for $t_{c}$. For the impingement of water droplet on aeroengine fan blades, $C_{1}$ is approximately an order of magnitude greater than $V$, 4, thus:

$$
t_{c}=\frac{V}{4 a\left(C_{1}^{2}-V^{2}\right)} \approx \frac{V}{4 a C_{1}^{2}}
$$

For an axisymmetric impingement, the position of the release wave can be described by eqn. 15 [4.

$$
X_{r}=X_{c}-C_{1}\left(t-t_{c}\right)
$$

Thus, eqn. 15 can also be used for the jet-front described by a quadratic, where the expression for $X_{c}$ can be found by substituting eqn. 14 into eqn. 8 .

$$
X_{c}=\sqrt{\frac{V t_{c}}{a}} \approx \sqrt{\frac{V^{2}}{4 a^{2} C_{1}^{2}}}=\frac{V}{2 a C_{1}}
$$

The final expression required, $t_{r}$, is found by using the expression for $X_{r}$, eqn. 15. When $X_{r}=0$, $t=t_{r}$. Thus, if eqn. 15 is set to equal zero and rearranged, eqn. 17 is found.

$$
t_{r}=\sqrt{\frac{V t_{c}}{a C_{1}^{2}}}+t_{c}
$$

Now that all the expressions (for $X_{e}, X_{r}, t_{c}$, and $t_{r}$ ) have been found for both a quadratic rotated around its axis of symmetry and sphere of liquid impinging a planar surface, an expression for the impulse of the loading of the compressible stage for

\begin{tabular}{|c|c|c|}
\hline $\begin{array}{l}\text { Quan- } \\
\text { tity }\end{array}$ & Spherical droplet & $\begin{array}{l}\text { Jet quadratic- } \\
\text { front }\end{array}$ \\
\hline$X_{e}$ & Equation 3 & Equation 8 \\
\hline$X_{c}$ & $\operatorname{Vr} / C_{1}$ & Equation $\sqrt{16}$ \\
\hline$t_{c}$ & $r V / 2 C_{1}^{2}$ & Equation $\overline{14}$ \\
\hline$X_{r}$ & Equation 15 & Equation $\overline{15}$ \\
\hline$t_{r}$ & $3 r V / 2 C_{1}{ }^{2}$ & Equation \\
\hline
\end{tabular}
each can be calculated via eqn. 13 . Table 3 summarises the expressions that were substituted into eqn. 13 to give the values of impulse for sphere of
Table 3: Expressions substituted into eqn. 13

liquid $\left(J_{\text {droplet }}\right)$ and jet described by a polynomial $\left(J_{j e t}\right)$. These solved integrals, which have then been simplified, are shown below:

$$
\begin{gathered}
J_{\text {droplet }} \approx p \pi \frac{r^{3} V^{3}}{C_{1}^{4}}\left(\frac{7}{12}-\frac{V^{2}}{24 C_{1}{ }^{2}}\right) \\
J_{j e t} \approx p \pi\left(\frac{7 V^{3}}{96 a^{3} C_{1}^{4}}\right)
\end{gathered}
$$

A useful approximation to the expression for $J_{\text {droplet }}$ (eqn.18) can be made, using the same principle employed earlier. As $C_{1}$ is approximately an order of magnitude greater than $V$, one can ignore the second term in the expression, as this will be comparatively small. If this form of eqn. 18 and eqn. 19 are set equal to each other $\left(J_{\text {droplet }}=J_{\text {jet }}\right)$ and the equation simplified, the following relationship between $r$ and $a$ results:

$$
r \approx \frac{1}{2 a}
$$

Despite its simplicity, eqn. 20 provides a good 'equivalent' radius, based on the conservation of impulse imparted during the compressible stage. Figure 7 shows the expected temporal variation in force for a typical jet produced by the rig and by a droplet with the equivalent radius. As will be shown in the results section, typical values of impingement velocity and equivalent radius were $230 \mathrm{~ms}^{-1}$ and $2.5 \mathrm{~mm}$ (from a typical value of $a$ of 200) for the runs conducted. The temporal variation in the force, and thus the overall impulse, for both the jet with a curved-front described by a rotated quadratic and the spherical droplet are almost identical.

\section{Results}

\subsection{Characterisation of high-speed jets}

The frame rate $(500 \mathrm{kHz})$ of the ultra-high-speed recordings of the jet crossing the stand-off distance 


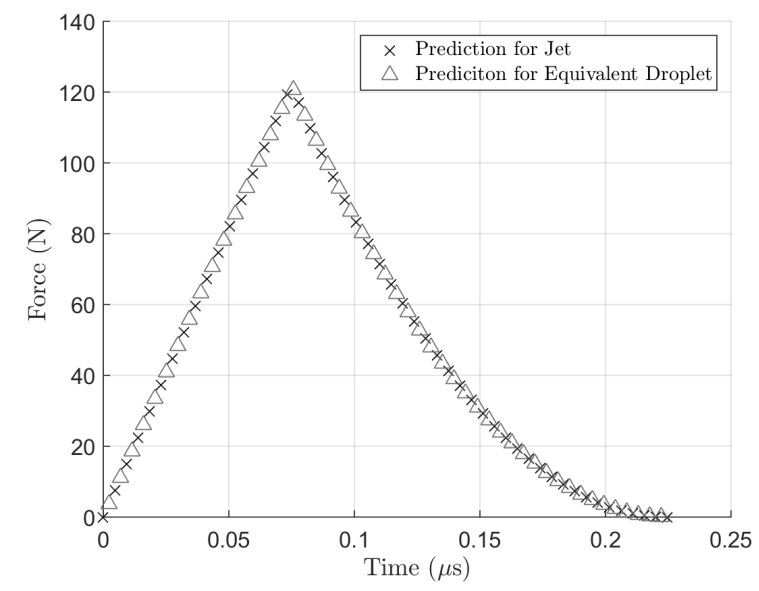

Figure 7: Comparison in temporal variation in force for jet and a droplet with the 'equivalent' radius

enabled the jet velocity and shape to be evaluated at an average (median value) of 24 positions for each run. With this volume of information, it has been possible to measure in detail the evolution of the jet as it traverses the stand-off distance. Figure 8 shows how the jet velocity increases with distance from the orifice. All runs show a similar trend of greater acceleration initially, with the rate of increase of velocity decreasing as the jet travels further. Impingement velocities (samples are approx. $10 \mathrm{~mm}$ from orifice) range from 210 to $250 \mathrm{~m} \mathrm{~s}^{-1}$.

Figure 9 shows how the curvature of the jet evolves across the stand-off, where eqn.20 has been used to calculate the equivalent radius. In this figure, the results from three runs, which do not fit the general trend, have been plotted using an alternative marker. For the majority of runs, there appears to be an initial period where the equivalent radius increases and then, after travelling approximately $3 \mathrm{~mm}$, the equivalent radius stabilises between $2 \mathrm{~mm}$ and $3 \mathrm{~mm}$. However, as the jet travels further the spread of values of equivalent radius increases, with the spread of values considerably tighter at a stand-off of $5 \mathrm{~mm}$ than $10 \mathrm{~mm}$.

\subsection{Impingement of planar specimens}

Figure 10 shows an example of the damage visible, under light microscopy, when a planar specimen of PMMA was impinged by a high-speed curved-fronted water-jet. An approximately circular central undamaged region is observed, with a deviation from the circular shape in the top right

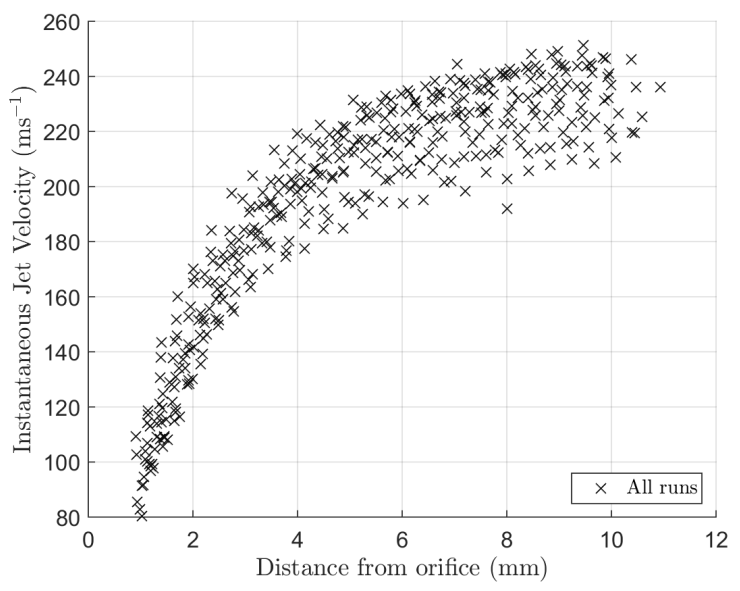

Figure 8: Variation in jet velocity across stand-off distance

of the micrograph. Other features include the short circumferential fractures beyond the main, approximately annular, region of disturbance to the surface. The images taken by the ultra-high-speed camera, and subsequent analysis, measured the impingement velocity of the jet to be $220 \mathrm{~ms}^{-1}$. The equivalent droplet radius was towards the larger end of the range produced, with a value of $3.7 \mathrm{~mm}$. This, in turn, lead to a prediction for the point where the shock front overtakes the contact periphery, $424 \mathrm{\mu m}$ from initial point of contact, to also be at the larger end of values expected. Equation 16 was used to make this prediction. This has been annotated on fig. 10 in the form of a circle with a radius equal to this predicted value.

Figure 11 shows the results of scanning the same damaged sample shown in fig. 10 with a $3 \mathrm{D}$ profilometer. An approximately annular depression is observed in the surface, with a deviation in the top right. In order to improve the clarity of the plastically deformed regions of the surface, the fullrange of heights measured has not been shown in fig. 11(a). Instead, a reduced range has been plotted, with white corresponding to values of height both greater than or equal to $0.5 \mu \mathrm{m}$, black to values less than or equal to $-0.5 \mu \mathrm{m}$. This is not the case in fig. 11(b) where the raw data has been plotted. The line AB (in fig. 11(a) was selected to bisect the annular depression. Figure 11(b) shows the two sides of the depression, with a depth of the order of hundreds of nm. The displacement across the central undeformed region, along AB, is $920 \mu \mathrm{m}$. 


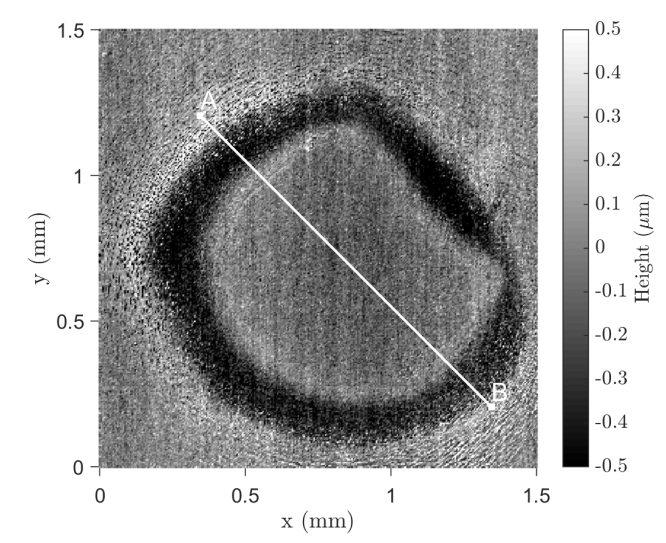

(a) 3D surface profile, any values of height greater than $0.5 \mu \mathrm{m}$ are shown as white and any values less than $-0.5 \mu \mathrm{m}$ as black.

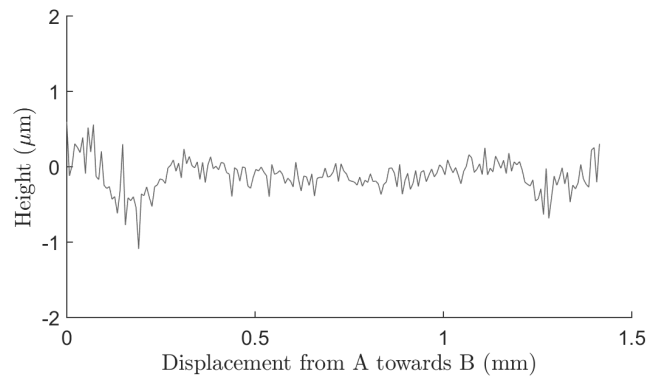

(b) Surface profile along line AB defined in fig. 11(a)

Figure 11: Surface profilometry measurement of sample shown in fig. 10

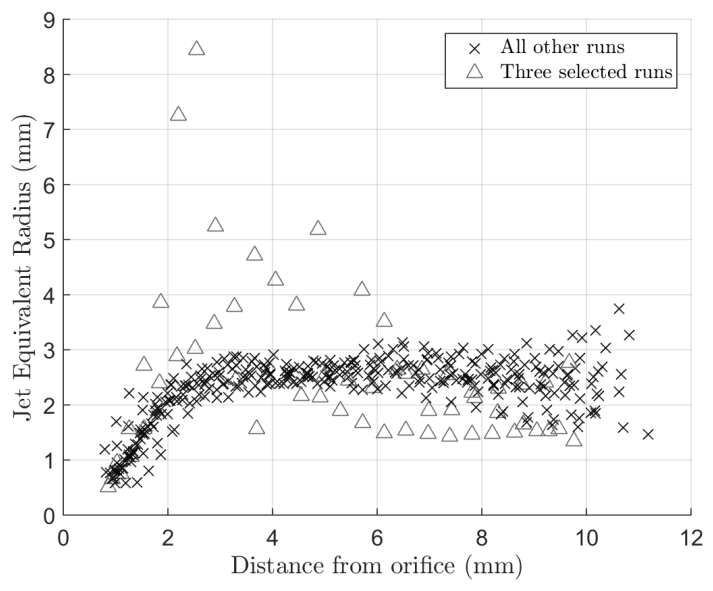

Figure 9: Variation in equivalent droplet radius across standoff distance

\subsection{Impingement of curved specimens}

Figure 12 shows a light micrograph of the damage observed on a curved specimen, with a $6 \mathrm{~mm}$ radius of curvature. Dark-field illumination was used in order to improved the visibility of the fractures. Although the same features are present (as observed on the planar surface), their shape differs significantly. The damage features are not symmetric around the central axis of the jet; they are in the form of an oval, as opposed to approximately circular form. The impingement velocity of the jet

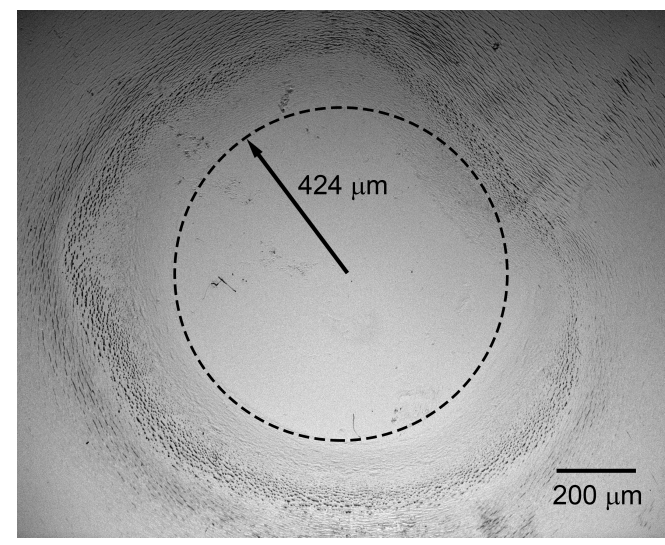

Figure 10: Light micrograph of damage caused to planar specimen impinged by jet at $220 \mathrm{~m} \mathrm{~s}^{-1}$ with equivalent radius $3.7 \mathrm{~mm}$. Shock front predicted to overtake contact periphery at $424 \mu \mathrm{m}$ from initial point of contact.

was slightly higher in this case (compared with the planar specimen previously described) at $240 \mathrm{~m} \mathrm{~s}^{-1}$. However, the droplet equivalent radius was significantly less, with a value of $1.9 \mathrm{~mm}$. Thus, the prediction for the point where the shock front overtakes the contact periphery on a planar surface is also less in this case; $229 \mu \mathrm{m}$ from initial point of contact. This has been annotated on this figure to allow comparison between a theoretical prediction that assumes surface form to be planar and the damage observed on samples with a radius of 


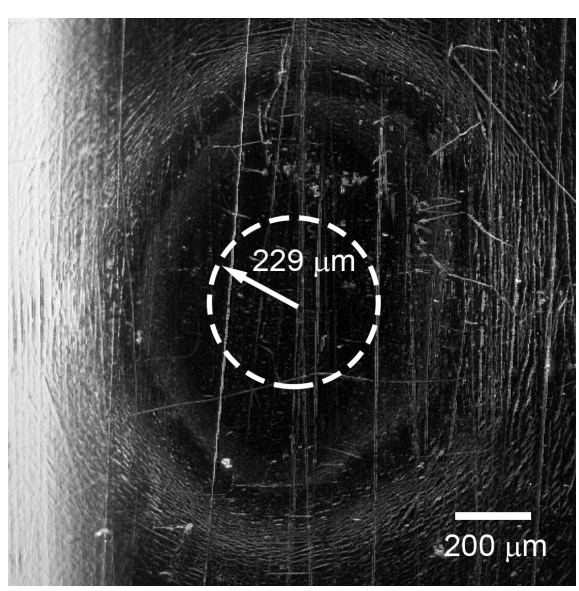

Figure 12: Light micrograph (using dark-field illumination) of a curved specimen, where plane of curvature is in horizontal direction with a $6 \mathrm{~mm}$ radius. The sample was impinged by jet at $240 \mathrm{~m} \mathrm{~s}^{-1}$ with equivalent radius $1.9 \mathrm{~mm}$. Shock front predicted to overtake contact periphery, if surface form assumed to be planar, at $229 \mu \mathrm{m}$ from initial point of contact.

curvature (in one plane).

Figure 13(a) shows the 3D profile of the sample shown in fig. 12. As with the features observed in the light micrograph (fig. 12), the annular depression remains, however, its shape has changed to an oval. As previously, in order to improve the clarity of the plastically deformed regions of the surface the full-range of heights measured has not been shown in fig $13(\mathrm{a})$ and a reduced range has been plotted (see figure caption for details). However, the removal of surface form was complicated by a slight 'rippling' on the surface of the PMMA rod, presumably due to small imperfections in the extrusion process. Thus, distinct vertical bands of the order of a few $\mu \mathrm{m}$ are observed around $x$ values of $0 \mathrm{~mm}$ and $1 \mathrm{~mm}$. It has not been possible thus far to remove this aspect of the original form and so the scale selected for fig. 12 is almost an order of magnitude greater than that for fig. 10. On initial viewing this might lead to the mistaken observation that the plastic deformation in the curved sample is smaller than for the planar. However, if fig. 11(b) is viewed (raw data, no reduced range), it can be seen that the depth of the depression is the same order as for the planar sample, hundreds of $\mathrm{nm}$.

Figure 14 shows light micrographs (using darkfield illumination) of typical damage observed as the radius of curvature of the surface was varied. Like the sample shown in fig. 12 the damage fea- tures have been transformed from a circular shape to an oval. Unfortunately, at radii of curvature below $3 \mathrm{~mm}$ it has not been possible thus far to image the damaged region of the surface. Therefore, the results of the tests conducted on samples with a 1 , $1.5,2$, or $2.5 \mathrm{~mm}$ radius of curvature have not been presented.

\section{Discussion}

\subsection{High-speed jet characterisation}

The results suggest, when selecting a stand-off distance there is a trade-off between achieving a sufficiently large jet velocity and having a sufficiently small spread of equivalent droplet radii. The stand-off distance selected, approx. $10 \mathrm{~mm}$, provided a good compromise between achieving a sufficiently high velocity to cause damage, whilst not being at too great a distance for an unacceptably large spread of equivalent droplet radii. As previously described, in the WDE of aeroengine fan blades, the expected droplet radius and radius of curvature of the leading-edge of a fan blade are approximately the same size. In this investigation, the range of equivalent droplet diameters upon impingement, $2 \mathrm{~mm}$ to $3 \mathrm{~mm}$, suggest that the range of surface curvatures tested, $1 \mathrm{~mm}$ to $7.5 \mathrm{~mm}$, were appropriate for the engineering context of this research. Finally, although the acceleration of the jets to the impingement velocity was repeatable, there were a few cases where the development of the jet curvature was not. This emphasises the need for characterisation of each impingement, to ensure the curved jet-front has formed successfully.

\subsection{Impingement of planar and curved surfaces}

As described in the introduction, our previous theoretical work 4] indicated, for the WDE of aeroengine fan blades, the form of the leading-edge (or curvature in one plane) affects the process of impingement and can not be assumed to be planar. The experimental results in this study, more specifically, the qualitative observations of the damage to the planar and curved specimens also suggest this to be the case. However, before this is discussed, it is worth exploring the level of confidence we can attribute to the results.

Firstly, the general structure of the damage, observed in this study, is the same as that observed previously with water droplets under very similar impingement conditions (see fig. 2). In both cases, 


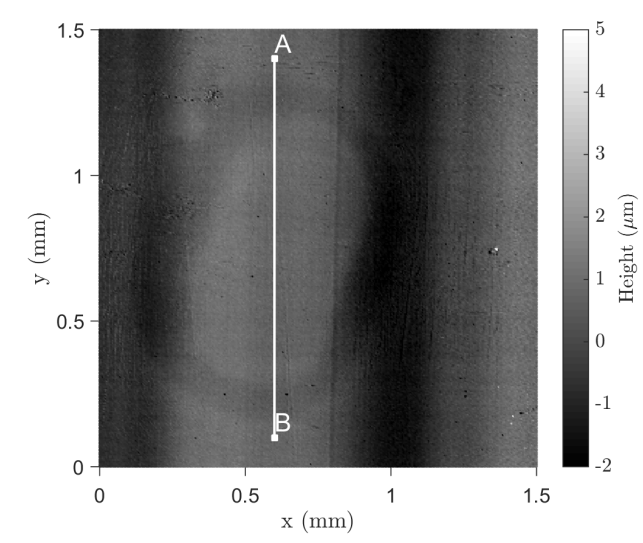

(a) 3D surface profile, any values of height greater than $5 \mu \mathrm{m}$ are shown as white and any values less than $-2 \mu \mathrm{m}$ as black.

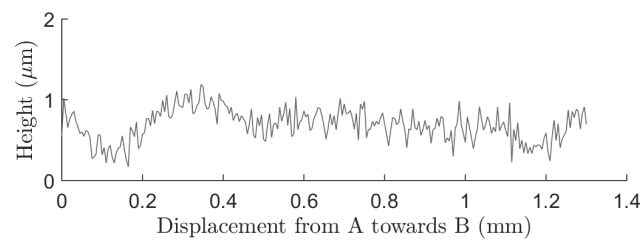

(b) Surface profile along line AB defined in fig. 13(a)

Figure 13: Surface profilometry measurement of sample shown in fig. 12

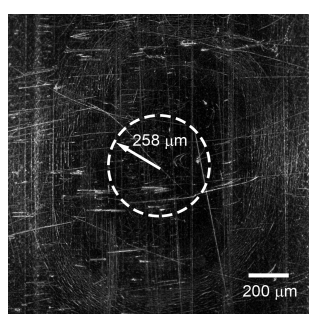

(a) $\mathrm{R}=7.5 \mathrm{~mm}$

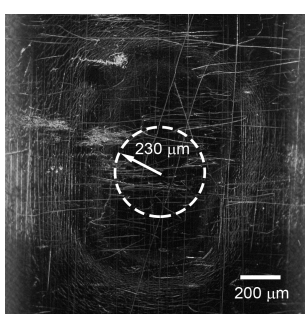

(b) $\mathrm{R}=6 \mathrm{~mm}$

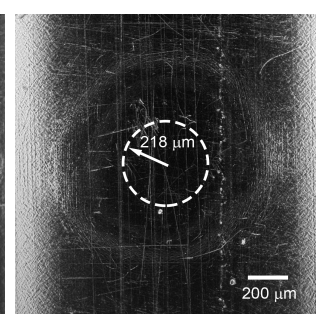

(c) $\mathrm{R}=5 \mathrm{~mm}$

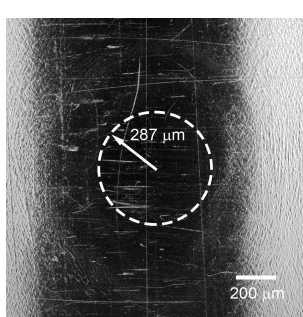

(d) $\mathrm{R}=4 \mathrm{~mm}$

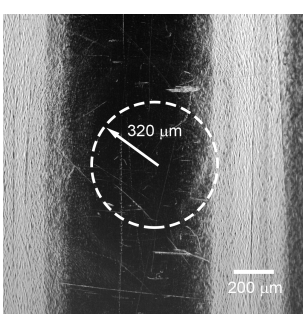

(e) $\mathrm{R}=3 \mathrm{~mm}$

Figure 14: Light micrographs (using dark-field illumination) of curved samples with decreasing radius of curvature $(R)$. Plane of curvature is in horizontal direction for all. Prediction for point where shock front expected to overtake contact periphery (based on direct measurement of impingement velocity and jet shape), if surface form assumed to be planar, is shown as an annotation in each case.

there is a central undamaged region, surrounded by an annular depression, which is then surrounded by short circumferential crazing fractures. It is also reassuring that the depth of deformation in both cases is of the same order (hundreds of $\mathrm{nm}$ ). Finally, the radius of the central undamaged region is expected to strongly correlate with the point at which the shock front overtakes the contact periphery $\left(X_{c}\right)$. For the planar PMMA sample shown in fig. 10. $X_{c}$ is predicted to be $424 \mu \mathrm{m}$, compared with the measured radius of the undamaged region of $460 \mu \mathrm{m}$.

In addition to the damage observed, the quantitative characterisation of each impingement is also a source of confidence. The developments in ultrahigh-speed digital imaging and it processing, have allowed this study to have a level of characterisa- tion of the incoming jets no previous study has been able to enjoy. This characterisation enabled the curved jet-front form to be described more accurately, as a rotated quadratic, for which the equivalent droplet was defined using an established theoretical approach ('Guided Acoustic Shock'). However, it should be noted that there is some evidence of asymmetry in the high-speed jets; on some impingements conducted on planar specimens the damage is not always perfectly circular (as is the case in fig. 10.

Given the above, it is hard to offer an alternative explanation, than change of surface form, for the significantly different shape of damage between the planar and curved samples. All the light micrographs have been annotated with the circle that 
should mark the edge of the central undamaged region if surface form can be neglected and assumed to be planar. However, a significant change in the geometry is visible on the curved samples; the circular form of the damage changes to an oval. This is consistent with our theoretical prediction 4, where the shock front is predicted to overtake the contact periphery earlier circumferentially (on the plane of curvature) than along the length of the leadingedge. If this theoretical prediction were not correct, a circular damage pattern on the surface of the curved samples, as indicated by the annotations on the figures, would be expected. Yet, the results suggest that this is not the case and that the form of the surface is significantly affecting the impingement.

\subsection{Implications for WDE of aeroengine fan blades}

Unlike many other studies investigating WDE, in this work, the volume of material loss has not been calculated, nor the microstructual aspects of the response of the material tested (PMMA) explored indepth. This is because, although interesting, these activities would be peripheral to the central purpose of this study. The purpose of using PMMA was to explore the effect of surface form, more specifically curvature (in one plane), on an individual highspeed impingement, using a material where the response is measurable and well-understood. Why? Because when the theory was extended [4] to include a radius of curvature it suggested the loading of an individual high-speed impingement would be significantly different, ergo, potentially a significantly difference process of WDE. However, this had never been confirmed experimentally.

This study provides qualitative experimental confirmation of the theory, but what does the mean for the process of wear; the mechanisms of WDE? This is a question that is likely to take a number of further studies to answer, however the theory provides insight. The reason why the surface curvature effects the high-speed impingement is, due the change in geometry, the shock front in the water does not overtake at all points on the contact periphery in unison, as would be the case for a planar surface; it occurs first on the plane of most curvature (around the leading-edge) and last along the plane of least (along the length of the leading-edge). This has implications for all four general mechanisms of WDE. Both direct deformation and stress wave propagation are now the result of an asymmetric loading through a reduced cross-sectional area.
Lateral outflow jetting is likely to be more severe around, than along the length of, the leading-edge. Finally, hydraulic penetration, which is dependent on the previous three mechanisms to change the surface profile sufficiently to become active, is now likely to be different due to a new set of initial conditions and changed surface geometry to erode.

WDE is a dynamic and complex process of wear, yet it is plausible that an asymmetric impingement process is likely to lead to an asymmetric process of WDE. This asymmetry may lead to increased erosion occurring around, than along the length of, the leading-edge and that material and surface properties in the plane of greatest curvature are of increased importance in determining erosion resistance during the early stages of WDE for aeroengine fan blades. These ideas and their investigation are the next steps of this work.

\section{Conclusions}

An established experimental method, using novel characterisation provided by ultra-high-speed imagining, has been used to investigate the effect of form on a high-speed droplet impingement. This is an essential precursor to developing a more complete understanding of how the form of the leading-edge influences the Water Droplet Erosion of aeroengine fan blades. The main conclusions were:

- The high-speed curved-fronted water-jets are accurately experimentally recreating a highspeed water droplet impingement.

- Ultra-high-speed digital imaging allows these impingements to be characterised with an accuracy not previously possible.

- The curvature of the surface (in one plane) significantly influences a high-speed droplet impingement, in a manner qualitatively consistent with the theoretical predictions made. This is likely to have significant implications for the WDE that occurs as a result.

The results shown here offer strong evidence in support of theoretical predictions made, yet they do not yet constitute a validation. For this to be achieved, quantitative comparison is required. This has started but is not yet complete and more results are required before this can be shared. However, the first steps in verifying the theoretical prediction have been made and more complete theory of WDE for aeroengine fan blades is emerging. 


\section{Nomenclature}

$a$ Coefficient of second-order term in quadratic function describing jet fron

A Position of droplet/jet periphery (m)

B Position of solid surface (m)

C Position of contact periphery $(\mathrm{m})$

$C_{1} \quad$ Shock velocity in liquid droplet/jet $\left(\mathrm{m} \mathrm{s}^{-1}\right)$

$F \quad$ Force $(\mathrm{N})$

$\hat{\mathbf{i}} \quad$ Unit vector in $\mathrm{x}$-direction $(\mathrm{m})$

$\hat{\mathbf{j}} \quad$ Unit vector in $\mathrm{y}$-direction $(\mathrm{m})$

$J \quad$ Impulse $(\mathrm{Ns})$

$J_{\text {droplet }}$ Impulse due to droplet impingement $(\mathrm{Ns})$

$J_{\text {jet }} \quad$ Impulse due to jet impingement $(\mathrm{Ns})$

$J \quad$ Impulse $(\mathrm{Ns})$

$p \quad$ Pressure $\left(\mathrm{N} \mathrm{m}^{-2}\right)$

$\rho_{1} \quad$ Density of liquid droplet/jet $\left(\mathrm{kg} \mathrm{m}^{-3}\right)$

$r \quad$ Radius of liquid droplet/equivalent radius of jet $(\mathrm{m})$

$R \quad$ Radius of curvature (in one plane) of solid surface $(\mathrm{m})$

$t \quad$ Time from start of impingement (s)

$t_{c} \quad$ Time at which shock front overtakes contact periphery $(\mathrm{s})$

$t_{r} \quad$ Time at which pressure release wave reaches central axis; duration of compressible stage (s)

$V \quad$ Impingement velocity $\left(\mathrm{m} \mathrm{s}^{-1}\right)$

$X_{c} \quad$ Position of contact periphery when $t=t_{c}$ (m)

$X_{e} \quad$ Position of contact periphery relative to central axis $(\mathrm{m})$

$X_{r} \quad$ Position of pressure release wave head (m)

\section{Acknowledgements}

The authors are very grateful to EPSRC for sponsoring the $\mathrm{PhD}$ studentship, ref. 1685710, that allowed this research to take place. We have been very fortunate to benefit from the expertise of Prof Fabrice Pierron and Dr Lloyd Fletcher (both of University of Southampton) with regards to the use of ultra-high-speed imagining. The camera used, a Shimadzu HPV-X2, is a result of Prof Pierron's EPSRC Established Career Fellowship (www.photodyn.org), ref. EP/L026910/1. We are also very grateful to Lawrence Miles (of University of
Southampton) for the development of the Ardinuo microcontroller, which triggered the flash and camera, and to Adrian Walker (of Safire Associates, Southampton), for the detailed design and manufacturing of the experimental rig.

\section{Declarations}

On behalf of all authors, the corresponding author states there is no conflict of interest.

\section{References}

[1] A. I. Sayma, M. Kim, N. H. S. Smith, Leading-edge shape and aeroengine fan blade performance, Journal of Propulsion and Power 19 (2003) 517-520.

[2] O. G. Engel, Damage produced by high-speed liquiddrop impacts, Journal of Applied Physics 44 (1973) 692-704.

[3] J. E. Field, Elsi conference: invited lecture. liquid impact: theory, experiment, applications, Wear 233 (1999) $1-12$.

[4] C. B. Burson-Thomas, R. Wellman, T. J. Harvey, R. J. K. Wood, Importance of surface curvature in modeling droplet impingement on fan blades, Journal of Engineering for Gas Turbines and Power 141 (2018) 031005-031005-9.

[5] A. K. Gujba, Z. Ren, Y. Dong, C. Ye, M. Medraj, Effect of ultrasonic nanocrystalline surface modification on the water droplet erosion performance of ti6al4v, Surface and Coatings Technology 307 (2016) $157-170$.

[6] ASTM International, Astm g40-15, standard terminology relating to wear and erosion, 2013. URL: www astm. org

[7] W. F. Adler, Rain impact retrospective and vision for the future, Wear 233 (1999) 25-38.

[8] W. F. Adler, The Mechanics of Liquid Impact, volume 16, New York: Academic Press, 1979, pp. 127-178.

[9] M. Lesser, Thirty years of liquid impact research: a tutorial review, Wear 186-187 (1995) 28-34.

[10] F. J. Heymann, Liquid Impingement Erosion, volume 18, ASM International, Materials Park, Ohio, 1992, pp. 221-231.

[11] F. P. Bowden, J. E. Field, The brittle fracture of solids by liquid impact, by solid impact, and by shock, Proceedings of the Royal Society of London. Series A, Mathematical and Physical Sciences 282 (1964) 331-352.

[12] J. E. Field, J. P. Dear, J. E. Ogren, The effects of target compliance on liquid drop impact, Journal of Applied Physics 65 (1989) 533.

[13] J. H. Brunton, M. C. Rochester, Erosion of Solid Surfaces by the Impact of Liquid Drops, volume 16, New York : Academic Press, 1979, pp. 185-244.

[14] N. K. Bourne, T. Obara, J. E. Field, High-speed photography and stress gauge studies of jet impact upon surfaces, Philosophical Transactions of the Royal Society of London. Series A: Mathematical, Physical and Engineering Sciences 355 (1997) 607-623. 
[15] G. P. Thomas, J. H. Brunton, Drop impingement erosion of metals, Proceedings of the Royal Society of London. Series A, Mathematical and Physical Sciences 314 (1970) 549-565.

[16] W. F. Adler, R. F. Vyhnal, Rain erosion of ti-6al-4v, in: A. A. Fyall, R. B. King (Eds.), 4th Int. Conf. Rain Erosion Assoc. Phenom., 1974, pp. 539-569.

[17] O. G. Engel, Waterdrop collisions with solid surfaces, Journal of Research of the National Bureau of Standards 54 (1955) 281-298.

[18] A. A. Fyall, Single impact studies with liquids and solids, in: A. A. Fyall, R. B. King (Eds.), 2nd International Conference on Rain Erosion and Associated Phenomena, volume 2, RAE Farnborough, UK, 1967, pp. 563-586.

[19] W. F. Adler, S. V. Hooker, Rain erosion behaviour of polymethylmethacrylate, Journal of Materials Science 13 (1978) 1015-1025.

[20] J. Field, D. Gorham, D. Richerby, High-Speed Liquid Jet and Drop Impact on Brittle Targets, American Society for Testing and Materials, Philadelphia, PA, 1979, pp. 298-319.

[21] W. F. Adler, Single water drop impacts on polymethylmethacrylate, in: J. E. Field (Ed.), 5th Int. Conf. on Erosion by Liquid and Solid Impact, Cavendish Laboratory, University of Cambridge, 1979, p. Paper 9.

[22] W. F. Adler, Rain erosion testing, Proc. SPIE 1112 (1989) 275-294.

[23] R. J. Hand, J. E. Field, D. Townsend, The use of liquid jets to simulate angled drop impact, Journal of Applied Physics 70 (1991) 7111-7118.

[24] F. P. Bowden, J. H. Brunton, The deformation of solids by liquid impact at supersonic speeds, Philosophical Transactions of the Royal Society of London. Series A: Mathematical, Physical and Engineering Sciences 263 (1961) 433-450.

[25] Y. K. Hong, K. H. Moon, Experimental research on a waterjet to simulate erosion by impact of a water drop, Wear 368-369 (2016) 116-123.

[26] Perspex cell cast acrylic; technical data sheet, https://www.theplasticshop.co.uk/plastic_ technical_data_sheets/perspex_technical_ properties_data_sheet.pdf 2018 . Accessed: 2018-09-27.

[27] Mechanical properties; extruded acrylic rod (clear), https://www.theplasticshop. co.uk/plastic_technical_data_sheets/ extruded-acrylic-rod-mechanical-properties.pdf, 2018. Accessed: 2018-09-27.

[28] A. Trujillo-Pino, K. Krissian, M. Alemn-Flores, D. Santana-Cedrs, Accurate subpixel edge location based on partial area effect, Image and Vision Computing 31 (2013) $72-90$.

[29] Q. Zhou, N. Li, X. Chen, T. Xu, S. Hui, D. Zhang, Analysis of water drop erosion on turbine blades based on a nonlinear liquid-solid impact model, International Journal of Impact Engineering 36 (2009) 1156-1171.

[30] K. K. Haller, Y. Ventikos, D. Poulikakos, P. Monkewitz, Computational study of high-speed liquid droplet impact, Journal of Applied Physics 92 (2002) 2821-2828.

[31] M. B. Lesser, J. E. Field, The impact of compressible liquids, Annual Review of Fluid Mechanics 15 (1983) 97-122.

[32] S. S. Cook, Erosion by water-hammer, Proceedings of the Royal Society of London. Series A, Containing
Papers of a Mathematical and Physical Character 119 (1928) 481-488. 